\title{
Materials used in mechanical heart valves: A geometrical perspective
}

\author{
Abhimanyu Tharayil, ${ }^{1}$ Vivek Verma, ${ }^{1}$ Malay K. Das, ${ }^{2}$ and Kamal K. Kar ${ }^{2,3 *}$ \\ ${ }^{1}$ Department of Materials Science and Engineering, Indian Institute of Technology Kanpur, Kanpur- \\ 208016, UP, India \\ ${ }^{2}$ Department of Mechanical Engineering, Indian Institute of Technology Kanpur, Kanpur-208016, \\ India \\ ${ }^{3}$ Advanced Nanoengineering Materials laboratory, Materials Science Programme, Indian Institute of \\ Technology Kanpur, Kanpur-208016, India \\ *Email: kamalkk@iitk.ac.in
}

The heart is one of the most vital organs in the human body and when the natural heart valves cannot perform its intended function effectively and efficiently it has to be repaired or replaced with artificial heart valves. Replacement valves are of two types: bioprosthetic or tissue valves and mechanical heart valves. The latter have long-term durability but lifelong anticoagulation is required with high risk of thrombosis. Biological valves are usually constructed from pig or cow tissues and are specially treated so that the body does not reject them. However they have a smaller lifetime comparable to their mechanical counterparts [1].

The ideal heart valve is required to have high thromboresistance, durability, easiness of attachment and should not cause (i) turbulence, (ii) blood trauma and (iii) noise. Unfortunately, artificial heart valves pertaining to the abovementioned properties do not exist and the currently available prosthetics have their own limitations. The choice and geometry of the material plays a very important role, as it should be able to withstand the harsh and corrosive environment of the body and should not lead to rejection. The flow variability within the mechanical valve is directly related to its morphology. The main loading mechanisms that happen in a heart valve are flexural, shear and tension in nature. The heart valves when working under pressure has a tendency to change the morphology of the leaflets and struts and thereby changing their mechanical properties. Currently, there are three main classes of mechanical valves (i) caged-ball, (ii) single leaflet or tilting-disk, and (iii) bileaflet valves. They are all comprised of three main elements: a closure mechanism, housing, and sewing ring. The development of Mechanical valves with respect to design has long graduated from the ball in cage valve to the tilting disc and to the longest serving bileaflet pyrolytic carbon valves. Figure 1 [2] gives the timeline of advent of different geometries of mechanical valves. The advantage with the ball in cage valve was the limited moving part but it had a high rate of thromboembolism which possessed a serious disadvantage. The tilting disc valve had lower rate of thromboembolism, but had major structural drawbacks like the fracturing of the struts which eventually led to its ousting from the international market, even though it is still used in countries like India. The Bi-Leaflet valve is the most commonly used mechanical valve owing to its improved hemodynamics and durability, but is still not devoid of the risk for thrombosis and is quite expensive.

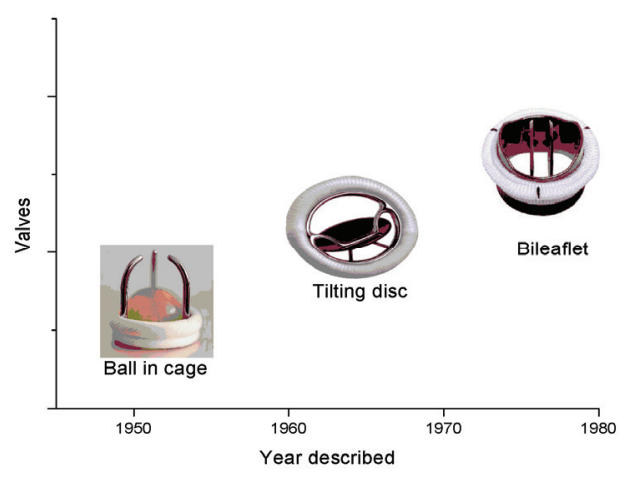

Figure 1: Mechanical valves through years

\section{References}

1. Yacoub MH, Cohn LH. Circulation 2004; 109:942-950

2. Richard A. DeWall, MD, Naureen Qasim, MD, and Liz Carr, BFA Ann Thorac Surg 2000;69:1 61221

3. http://www.pages.drexel.edu 